\title{
The Design and Implementation of Dynamic Time History Analysis on Architectural Structure CAD System
}

\author{
Qiu Lin \\ Nanchang Key Laboratory of material and structure detection Jiangxi University of Technology
}

\begin{abstract}
Keywords: Architecture Structure; Dynamic Time History Analysis; Newmark $-\beta$ Method; Computer Aided Design
\end{abstract}

\begin{abstract}
This paper firstly elaborates aseismic design theory and the development as well as research condition of CAD to briefly explain the function and necessity of dynamic time history analysis in architectural structure design. Then, it comes up with particular views for the advancement of architecture structure $\mathrm{CAD}$ system which is also the aim of this paper. The architecture structure CAD system application can be regarded as the realization of pre processing and post processing of SDY. Users can set the specific parameters dynamic time history analysis, choose and formulate seismic wave to carry out the calculation. In addition, the result of post-processing window can be shown with text and graphical representation. Besides, this paper also represents the application of dynamic time history analysis in two real projects. The research result can be directly adopted in aseismic calculation of architecture structure and to optimize the performance of architecture structure CAD. Meanwhile, this paper provides a solid foundation to develop and improve the calculation method of the architecture aseismatic design.
\end{abstract}

\section{Introduction}

With the rapid development of science and technology, scientific computing work becomes heavy with a sky rocketing number of data which needs to be analyzed. The visual approach can help researchers quickly acquire more information and directly have a good knowled ge of the result. CAD (Computer Aided Design computer-aided design) appeared in 1960s applied the high-speed computing power, mass storage, data processing ability, artificial intelligence, and computer graphics so as to organically combines comprehensive analysis ability, making it play a tremendous role. What's more, it can also serve various fields. In the process of architectural design, the application and usage of architecture CAD software have brought great convenience to design work.

Architectural CAD started late in China. In 1980s, relevant institutions, colleges and universities began to develop the framework of a computer aided design system. These earlier ACD systems have been greatly improved comparing to artificial design yet with obvious demerits: slow speed, poor quality of the drawing, poor generality etc, which have a large gap with developed countries. It was until the beginning of 1990s that software engineers developed system software with graphic interactive capabilities based on the pure mechanical analysis calculation software. In recent years, the architectural CAD development shows a flourishing situation. Some software will not only meet the structure design requirement of designing institutes but also has new editions with sophisticated 
functions and improved working efficiency. So far, engineering design industry is the one with mature CAD application.

At present, computer-aided design technology has been developed quickly and the CAD system has been perfected. However, CAD systems for some special structures (such as complex and high-rise structures) lag behind the general application software. In recent years, with the rapid social and economic development, the construction industry has been developed rapidly and there is a sky rocketing number of high-rise buildings as well as complicated constructions. Even though, there is some relevant calculation software, which cannot meet the needs of users along with the development of architecture.

Previous building structure CAD software only analyzes earthquake response spectrum to calculate vibration as well as stressed conditions, which will lead to uniformity and non-locality of seismic analysis. That is to say, choosing the same seismic grade with site classification, the result shall be same regardless of the location of the architecture. In contrast, the dynamic time history analysis method overcomes this limitation and users can input seismic wave based on the geological survey result and earthquake record. Different seismic waves will ignite different effects and damages on the same architecture. Therefore, analysis on time history dynamic response is necessary for architecture structures with requirements for seismic resistance, especially for high-rise buildings.

\section{Contents}

Structural design and dynamic time history analysis: With the development of computational mechanics and modern architectural design, human beings have accumulated a glittering array of knowledge about earthquake damage on building. What's more, people also have a deeper understanding on the dynamic behavior of building structure, which stimulates the development of theory in building structures seismic analysis.

Over several decades, the theory in building structures seismic analysis basically undergoes three stages of static analysis, response spectra analysis and dynamic analysis.

Static method:

Static method is the most commonly used analysis method. It assumes that structure and site have same vibration, takes the horizontal seismic inertial force $\mathrm{F}$ as the static force applied to buildings which is equal to gravity structure loading $G$ multiplying seismic coefficient $\mathrm{k}$, namely, $\mathrm{F}=\mathrm{kG}$. While carrying out seismic checking, it ignores dynamic characteristics of structures while taking earthquake acceleration as the single factor for earthquake damaged structure with great limits, which is only adapted to structure with high stiffness.

Response spectrum method:

Response spectrum method is pseudo-static method, which determines the equivalent seismic loads based on the accelerated response spectrum curve and carries out structure calculation analysis with static method. The maximum horizontal base shear is equal to $\mathrm{Fk}(\mathrm{T}) \mathrm{G}$, among which $\mathrm{k}$ ( T) refers to the specific value of acceleration response spectrum and maximum acceleration.

Response spectrum method has made greater advancement comparing to the static method, which changes the assumption and can truly reflect the vibration characteristics with simple ideas and calculation method. However, it does not take the effect of earthquake motion's time duration into consideration, which still takes earthquake inertia force as the static force.

Dynamic method: 
Dynamic method, which is also known as the dynamic time-history response analysis method, applying earthquake and artificial waves to structure and directly carrying out integration so as to know the analysis method of any time. In the course of dynamic analysis, there are concrete and clear requirement on the input motion, dynamic mockup of structure and component, time history analysis method and design principle. It can help us to have more reliable design based on detailed calculations.

At present, there is some architecture structure software for high-rise building both at home and abroad, which is an integrated system, or professional CAD software with special characteristics which can meet the design needs for high-rise buildings to some degrees. However, there are demerits for the existing architecture design software. In terms of interface and function, the software is relatively perfect in foreign countries. Yet, the construction habits and regulations are different, so only by transformation, can we make those be adapted to Chinese designers. Domestic software specifications and standards are closely linked to China, but in certain aspects the following defects still exist:

Firstly, the structural analysis theories are limited which will affect the ability to analyze complicated structure as well as the accuracy.

Secondly, the automatic optimization function is not perfect and is less precise.

Thirdly, the system of structure design is not perfect because the analysis and design (such as cutting plan, water-floating and curbing) are not enough, which indicate that the design software cannot systematically complete the design task which make domestic software drop behind foreign design software.

Fourthly, the standard node base is small, lacking components which can help to maintain the system such as standard node and wrap angle etc.

Fifthly, the domestic post processing ability is poor with weak performance in terms of automatically generating engineering drawings, which cannot form management document and the intelligent transformation of CAD and CAM.

Dynamic time history analysis and design in the architecture structure. We get the following kinematics equations formulate of buildings under the environment of earthquake based on the finite element analysis

$$
\mathrm{M} \ddot{X}(t)+C \dot{X}(t)+K X(t)+K X(t)=-M \ddot{Z}(t)
$$

Among which

$\ddot{X}(\mathrm{t}) 、 \dot{X}(\mathrm{t}) 、 \mathrm{X}(\mathrm{t})$ respectively refers to accelerated matrix, speed matrix and shifting matrix.

$\ddot{Z}(t)$ is the ground motion accelerated matrix.

If the total number of a building is $\mathrm{n}$ and the shifting of every floor is $X_{i}(t)$, then we can get the following formulate: 


$$
X(t)=\left\{\begin{array}{c}
X_{1}(t) \\
X_{2}(t) \\
X_{3}(t) \\
\vdots \\
X_{n}(t)
\end{array}\right\}
$$

$$
\ddot{X}(t)=\left\{\begin{array}{c}
\ddot{X}_{1}(t) \\
\ddot{X}_{2}(t) \\
\ddot{X}_{3}(t) \\
\vdots \\
\ddot{X}_{n}(t) \\
\end{array}\right\}
$$

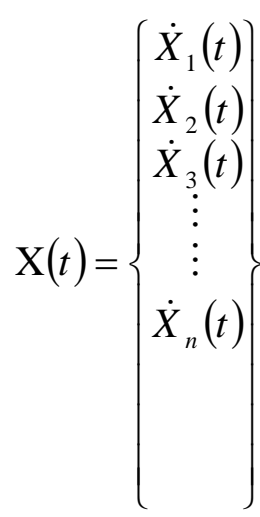

$\ddot{Z}(t)=\left\{\begin{array}{c}\ddot{Z}_{1}(t) \\ \ddot{Z}_{2}(t) \\ \ddot{Z}_{3}(t) \\ \vdots \\ \vdots \\ \ddot{Z}_{n}(t)\end{array}\right\}$

The accelerated speed of earthquake in every floor is same,

$\mathrm{M}$-structure coordination quality matrix: Supposing $\mathrm{M}$ is the floor quality and other elements are O.

K-structure stiffness matrix, each element represents the interactive relation of the line level and row level. When there is a shifting, they shall interact with each other.

C-structure damping matrix, which is also known as coordination matrix: supposing the ratio of damping force and velocity of particle motion in the course of vibration, and in general the damping force is in proportion to mass matrix and stiffness matrix and the data depends on the structure vibration frequencies. In architecture structure CAD implementation the key point to carry out dynamic time history analys is is effectively and accurately figuring out the equilibrium equation of structural dynamic response.

The application and realization of dynamic time history analysis: In early times of implementing architecture CAD, there is not a strong connection between the destroy of low layers and the structure without the function of dynamic time history analysis, which provides reliable data for the reasonable construction of high-rise buildings with seismic performance, What's more, the visualization function in architecture $\mathrm{CAD}$ can help relevant workers to understand and analyze the calculation result and assist designers choose, layout the structure as well as carry out force analysis.

For example, in the overall CAD structure system, the functions of sub-modules show as follows:

Firstly, the graphics input module: it refers to the window for users to input building architecture, and the procedure firstly defines relevant information among layers (such as high layer). Then, it defines the spatial stress structure (such as pillar, stress structure, floor), component information (such as size and strength etc) as well as loading. Based on this, users can determine the architecture structure based on human-computer interaction and transfer it into text information. 
Secondly, mechanical calculation module: that is to carry out mechanical analysis of the generated information, including calculating three dimensional element procedure SSW and thin wall trussing procedure 55, floor, secondary girder and brick calculation.

Thirdly, structural working drawing module: based on the above result, the procedure automatically generates working drawing and users can modify the working drawing.

Fourthly, basic module: which is adopted in building foundation design.

Fifthly, dynamic time history: it aims to analyze and calculate the response and result input of building in the effect of various seismic waves.

Sixthly, plane stress module: it refers to the component internal force, transformation finite element analysis and result input.

Seventhly, budget module, it is used to calculate the general budget.

Eighthly, visualization module: it aims to make the graphics be visual.

The CAD dynamic time history analysis can serve the architecture structure based on the following steps:

Firstly, based on the graphic input system, we can determine the architecture structure based on the human-computer interaction and transfer the graphic information into data information.

Then, based on floor and secondary girder calculation as well as SSW, we can carry out mechanical analysis on the data information so as to make the nature of integrity and each component close the reality such as stiffness, shifting and internal force.

Under the effect of specific regional condition, earthquake intensity and seismic wave, the architecture shall have forced vibration based on the mechanical characteristics. Besides, the shifting and stress can be calculated during the vibration time by SDY.

Take the maximum value of shifting, reaction force, shearing force and bending moment during the vibration time and then compare with the result calculated by CQC. Users can directly find the weak floor and the shifting floors as well as the floors with the stress more than the result calculated by CQC.

Users can analyze the result by ADY to judge whether the architecture can resist the force caused by the seismic wave or not so as to judge the vibration effect. For example, in the shifting analysis and stress results, we can find out that the vibration effect is poor and we have to re-enter the module and carry out modification on the structure design. We have to repeat the above four steps until the completion of dynamic time history analysis.

Sixthly, the procedure enters into the working drawing module to automatically generate the working drawing.

\section{Conclusions}

We have encountered with a glittering array of troubles in the course of carrying out dynamic time history analysis. For example, in the calculation selection we have tried many times. We have compared the central difference method, Runge-Kutta, Wlison, Newmark- $\beta \beta$, and finally select Newmark $\beta$ with a high degree of stability. What's more, we adopt Fortran which is convenient to carry out data calculation so as to make the procedure become more stable. In terms of data input and graphical display, we consult the requirement of designers and adopt SDY, which has been adopted in thousands of design institute, as well as complicated and high-rise buildings with 
and is widely accepted. Starting from the perspective of users, we design convenient, clear and quick graphic interface which greatly improves the working efficiency.

SDY has achieved great performance which remains to be developed. Besides, it has a great space for development in terms of calculation. At present, the calculation result is assumed based on flexibility which shall be developed toward the trend of elastoplasticity so as to make the calculation result be closer to the reality. The calculation result does not only show in the graphic pattern, the component as well as reinforcement also shows in working drawing presentations. Based on the above efforts, the dynamic time history analysis result can be directly applied to the structure construction, which is the development trend. We hope that the dynamic time history analysis can be fully analyzed and perfected in the near future.

\section{Acknowledgment}

This work was supported by Project on professional and characteristical construction of Jiangxi province 2010 (Civil Engineering) and Project on the planning and construction of disciplines in Jiangxi University of Technology (Structure Engineering)

\section{References}

[1] Lysaght P, Blodget B, Mason J, et al. Invited paper: Enhanced architectures, design methodologies and cad tools for dynamic reconfiguration of xilinx fpgas[C]//Field Programmable Logic and Applications, 2006. FPL'06. International Conference on. IEEE, 2006: 1-6.

[2] Luckham D C, Kenney J J, Augustin L M, et al. Specification and analysis of system architecture using Rapide[J]. Software Engineering, IEEE Transactions on, 1995, 21(4): 336-354.

[3] Benini L, Bogliolo A, De Micheli G. A survey of design techniques for system-level dynamic power management[J]. Very Large Scale Integration (VLSI) Systems, IEEE Transactions on, 2000, 8(3): 299-316.

[4] Rosenman M, Wang F. A component agent based open CAD system for collaborative design[J]. Automation in Construction, 2001, 10(4): 383-397.

[5] Wolf W, Jerraya A A, Martin G. Multiprocessor system-on-chip (MPSoC) technology[J]. Computer-Aided Design of Integrated Circuits and Systems, IEEE Transactions on, 2008, 27(10): 1701-1713.

[6] Requicha A A G, Voelcker H B. Solid Modeling: A Historical Summary and Contemporary Assessment[J]. IEEE Computer Graphics and Applications, 1982, 2(2): 9-24.

[7] Comer D E, Gries D, Mulder M C, et al. Computing as a discipline[J]. Communications of the ACM, 1989, 32(1): 9-23.

[8] Guarize R, Matos N A F, Sagrilo L V S, et al. Neural networks in the dynamic response analysis of slender marine structures[J]. Applied Ocean Research, 2007, 29(4): 191-198.

[9] Folegnani D, González A. Energy-effective issue logic[C]//ACM SIGARCH Computer Architecture News. ACM, 2001, 29(2): 230-239.

[10] Wayne S F, O'neil D A, Zimmerman C E, et al. Method and system for designing a cutting tool: U.S. Patent 5,377,116[P]. 1994-12-27. 